is a rare X-linked recessive disease. The MPS II is a hereditary metabolic disorder caused by accumulation of glycosaminoglycans (GAG) in organs and tissues due to mutations in the genes, which encode intralysosomal hydrolysis of macromolecules. Hunter syndrome is a progressive, multisystem disease. At the same time a child may have mental retardation and speech development delay, skeletal bone deformities, loss of vision, hearing loss.

Patients and Methods The study included 17 boys aged from 2 to 12 years old with genetically confirmed Hunter syndrome. All patients underwent examination by otolaryngologist, also tympanometry, diagnostic nasopharyngolaryngoscopy, registration of otoacoustic emission, audiometry, cardiorespiratory monitoring were performed.

Results The accumulation of GAG leads to a gradual narrowing of the nasopharynx and larynx lumen, a thickening of the tongue and vocal folds, laryngeal cartilages deformation, an enlarged of pharyngeal, palatine and lingual tonsils.

11 children $(64.7 \%)$ had chronic inflammation of the nasal mucosa and pharyngeal tonsil with frequent exacerbations. Pharyngeal tonsil hypertrophy was detected in 10 patients (58.8\%).

14 children $(82 \%)$ had a wide, thickened tongue. Hypertrophy of the tonsils was diagnosed in 8 children (47\%).

The voice had changed (hoarseness) in 16 children (94\%). Deformation of the epiglottis was detected in 4 people (23.5\%), tracheomalacia - in 1 child (5.8\%).

Among the complications acute sinusitis and exudative otitis media were detectable in $52.9 \%$ of patients. 10 children $(58.8 \%)$ had obstructive sleep apnea (OSA) syndrome, two of them $(11.7 \%)$ had a severe degree of the disease, which was an indication for adenotonsillectomy. Hearing loss was found in 10 boys $(58.8 \%)$.

In most patients $(82 \%)$ a combined pathology of the ear, throat and nose was detected, 2 children (11.7\%) had only adenoid hypertrophy, and only 1 child

$(5.8 \%)$ did not have any pathology of ENT organs.

Conclusion Functional disorders and diseases of the ear, throat and nose are found in most children with Hunter syndrome, which could be one of its early manifestations. An ENT specialist may suspect MPS type II according to the presence of an ENT pathology that is difficult to treat in a standard manner, combined with the pathology of other organs and systems.

Early diagnosis and the possibility of enzyme replacement therapy can control the disease progression and avoid early disability.

\section{HYPOKETOTIC HYPOGLYCEMIA AND HYPERAMMONEMIA AS PRESENTING FEATURES OF EARLY ONSET MULTIPLE ACYL-COA DEHYDROGENASE DEFICIENCY}

Vanja Ille*, Danijela Petković Ramadža, Tamara Žigman, Boris Filipović-Grčić, Ruža Grizelj, Dorotea Ninković, Dorotea Bartoniček, Ana Škaričić, Iva Bilandžija Kuš, Nikola Mesarić, Goran Krnjak, Ksenija Fumić, Ivo Barić. Department of Pediatrics, University Hospital Centre Zagreb, Zagreb, Croatia

\subsection{6/archdischild-2021-europaediatrics. 104}

Introduction Multiple acyl-coenzyme A dehydrogenase deficiency (MADD) or glutaric aciduria type II is a rare, autosomal recessive disorder of fatty acid and amino acid oxidation. Disease is caused by pathogenic mutations in ETFA or ETFB genes, which encode two subunits of electron transfer flavoprotein (ETF), or ETFDH gene, encoding for ETF-dehydrogenase. Phenotype is heterogenous, from severe neonatal acute metabolic decompensation, with or without congenital anomalies, to milder, late onset forms. Diagnosis is made by acylcarnitine and urinary organic acid analysis, and gene testing. Treatment is based on restricted fat and protein intake, high carbohydrate diet, with carnitine, riboflavin and CoQ10 supplementation.

Case Report Patient is a second child of unrelated parents. Family history unremarkable. Pregnancy was complicated by oligohydramnios. Delivery was on term, uneventful, BW 3740 $\mathrm{g}$, BL $53 \mathrm{~cm}$, AS 10,10. Tremor occurred as manifestation of hypoglycemia (BG $1.8 \mathrm{mmol} / \mathrm{l}$ ), corrected with 10\% oral glucose, on the first day of life. The newborn was euglycemic until 20 hours of life when irritability and somnolence were noticed in hypoglycemia (capillary glucose $2.3 \mathrm{mmol} / \mathrm{L}$, venous glucose was immeasurably low). Glucose infusion was started but patient deteriorated to bradypnea and bradycardia.

Cardiopulmonary reanimation lasted 40 minutes. Upon admission in NICU, patient was cardio-respiratory stable, but unconscious, hypertonic and soon developed seizures. Laboratory findings revealed metabolic acidosis ( $\mathrm{pH} 7.3, \mathrm{BE}-15.6)$, hyperlactatemia $(14 \mathrm{mmol} / \mathrm{L})$, hyperammonemia $(230 \mu \mathrm{mol} / \mathrm{L})$, increased aminotransferases and CK, normal blood glucose and positive urinary ketones. Brain MRI revealed cortical cytotoxic edema, restricted diffusion in corticospinal tracts and pons, and temporal atrophy. Ammonia normalized following high caloric intake, nitrogen scavengers, L-arginine, L-carnitine and vitamin B12. Urgent metabolic work-up revealed typical plasma acylcarnitine and urine organic acid profile for MADD. We started low protein and fat, high carbohydrate diet, continued with L-carnitine and added CoQ and riboflavin in therapy. Ultrasound showed mild hypertrophic cardiomyopathy and no malformations of other organs. Gene analysis revealed homozygous mutation c.1250C $>\mathrm{A}$ (p. Thr266Met) of the ETFA gene. In further course patient was stable, but with severe developmental delay, epilepsy and impaired vision.

Conclusion Beta oxidation defects, even with positive ketones, should be in differential diagnosis of neonatal hyperammonemia, as treatment is different than for other diseases. Lipid infusions, used to provide adequate caloric supply in other disorders, are contraindicated. Urgent metabolic work-up is mandatory for early diagnosis and optimal patient management. Clinical course with early acute cardio-respiratory failure, hypoglycemia and moderate hyperammonemia may be indicative for beta-oxidation defects.

\section{ALG6-CDG- CONGENITAL DISORDER OF GLYCOSYLATION WITH RECOGNIZABLE PHENOTYPE}

Marina Kokić Golub*, Tamara Žigman, Danijela Petković Ramadža, Maja Jurin, Duška Tješić Drinković, Stanko Ćavar, Sanja Džida, Jelena Nikolić, Andrija Miculinić, Ernest Bilić, Ksenija Fumić, Ivo Barić. Dom zdravlja Krapinsko-zagorske županije/Kliničko bolnički centar Zagreb

\subsection{6/archdischild-2021-europaediatrics. 105}

Congenital disorders of glycosylation (CDG) is a large group of genetic metabolic diseases that usually affects many different organ systems. Glycosylation is a complex process 
regulated by a numerous enzymes that modify and transfer sugar residues to amino acid side chains.

Over 150 different types of CDGs have been described. The most common are phosphomannomutase 2 (PMM2-CDG) and -1,3-glucosyltransferase deficiency (ALG6-CDG). Symptoms common to all CDGs are seizures, psychomotor delay, hypotonia, feeding disorders, liver disease and coagulopathy. Diagnosis is based on clinical presentation and sialotransferrin profiling and confirmed by gene analysis. Early diagnosis is critical in disorders for which specific therapy exists.

Female infant was born from a third, uneventful pregnancy. Parents are not related and older siblings are healthy. Since birth, the infant was severely hypotonic and exhibited developmental delay, feeding disorder and failure to thrive. At the age of three months she was transferred to our Department for further work-up. At that time, she had low body weight (4314 g, 1.c) and was hypotonic with reduced spontaneous movements. We had noticed dysmorphic features- hypertelorism, micrognathia, abnormal ears, flattened nose, rhizomelic limbs, inverted mamillae and abnormal fat distribution. She was fed with elemental infant formula through the nasogastric tube. The patient had small intestine malrotation that manifested with recurrent vomiting which was resolved after surgical intervention. She developed thrombosis of the brain venous sinuses due to coagulopathy (low concentration of antithrombin III, protein C and coagulation factors IX and $\mathrm{XI})$. She was also diagnosed with hypothyreosis and suffered from generalised oedema with hypoalbuminemia as a consequence of protein losing enteropathy, treated with albumin infusions. Protein losing enteropathy improved on elemental formula.

Severe epilepsy was drug-resistant. Sialotransferrin profiling pointed towards a glycosylation defect (elevated di-sialotransferrins, lowered penta- and tetra-sialotransferrins). Gene analysis revealed biallelic mutations in the ALG6 gene, which codes for glycosilation enzyme -1,3-glucosyltransferase.

ALG6-CDG has a recognizable phenotype characterised by hypotonia and proximal muscle weakness, extrapyramidal signs, pharmacoresistant epilepsy, coagulopathy, protein losing enteropathy and dysmorphic features. Described patients had poor prognosis. There is no specific therapy available. Early diagnosis is important to predict and treat symptoms and to stop diagnostic odyssey. It enables prenatal diagnostics in future pregnancies.

\section{DIVERSITY OF CLINICAL PHENOTYPE OF PATIENTS WITH PYRUVATE DEHYDROGENASE DEFICIENCY DUE TO PDHA1 GENE MUTATIONS}

Katarina Šikič ${ }^{*}$ Danijela Petković Ramadža, Tamara Žigman, Nina Barišić, Ivan Lehman, Johannes A Mayr, Holger Prokisch, Saskia B Wortmann, Wolfgang Sperl, Nikola Mesarić, Valentina Rahelić, Ksenija Fumić, David Ozretić, Maja Tomasović, Ivo Barić. Department of Pediatrics, University Hospital Centre Zagreb, Zagreb, Croatia

\subsection{6/archdischild-2021-europaediatrics. 106}

Pyruvate dehydrogenase (PDH) is a mitochondrial multiunit complex which catalyzes pyruvate oxidation (derived mainly from glucose) to acetyl-CoA which then enters tricarboxylic acid cycle. Its deficiency results in accumulation of lactate and alanine. Lactate/pyruvate ratio is normal. Brain is almost always affected because it obtains energy primarily from oxidation of glucose. PDH deficiency is most commonly due to mutation in an X-linked pyruvate dehydrogenase E1 alpha subunit gene (PDHA1). Diagnosis is a challenge especially in females not only because of variable clinical phenotype but also because of skewed X-chromosome inactivation - its pattern varies among individuals, but also among different tissues of the same individual. Therefore, measuring PDH activity is not a reliable guide to the diagnosis in females. Gene analysis may be used to confirm the diagnosis.

Causal treatment options include thiamine (cofactor of PDH), ketogenic diet and dichloroacetate. The goal of the study is to alert medical professionals to clinical variability of PDHC deficiency and thus make easier early diagnosis.

We present a spectrum of clinical phenotypes of six patients with PDH deficiency diagnosed and treated in our Center.

In patients 1 and 2 disease started antenatally - ultrasonography revealed brain ventriculomegaly. After birth MR demonstrated agenesis of corpus callosum, ventriculomegaly, cerebellar hypoplasia. Afterwards those patients had seizures and severe psychomotor retardation. Patient 3 had conatal hypotonia, respiratory difficulties and severe lactic acidosis. MRI revealed mild diffuse brain atrophy. At 8 years he has generalized hypotonia, walks with support. At age 6 months patient 4 presented with somnolence, metopic synostosis and mild white matter atrophy and ventriculomegaly. At 6 years she has hypotonia, moderate psychomotor retardation and ataxia. Patient 5 presented at 3 months with acute encephalopathy of unclear etiology. At 7 years she has isolated epilepsy and normal psychomotor development. Her older brother died at the age of 17 days due to cardiorespiratory failure of unknown cause. Patient 6 have had intermittent ataxia since 1 year and 8 months of age. Now at 10 years he has normal cognition. All patients had elevated plasma alanine and lactate (2,66-19,9 $\mathrm{mmol} / \mathrm{L}$; ref. range 0,63-2,40), and CSF lactate (3,64-8,52 $\mathrm{mmol} / \mathrm{L}$; ref. range 1,2-2,1), with normal lactate/ pyruvate ratio. All patients were on ketogenic diet and thiamine treatment. No patient had regression or deterioration while on therapy.

PDH deficiency should be in differential diagnosis of most neurological signs and symptoms. Early diagnosis may enable optimal treatment and proper genetic counseling.

\section{EARLY ONSET LIVER FAILURE DUE TO MITOCHONDRIAL DNA DEPLETION: CLINICAL COURSE OF FOUR PATIENTS}

Danijela Petković Ramadža*, Tamara Žigman, Ruža Grizelj, Dorotea Ninković, Lana Omerza Mirna Natalija Aničić, Marijana Ćorić, Johannes A Mayr, René Feichtinger, Saskia Wortmann, Holger Prokisch, Ksenija Fumić, Jurica Vuković, Ivo Barić. University Hospital Centre Zagreb and

\subsection{6/archdischild-2021-europaediatrics.107}

Mitochondrial DNA depletion syndromes (MDS) are a group of autosomal recessive disorders caused by disruption of mtDNA maintenance that results in reduced mtDNA content and disturbed energy production. MDS are genetically and phenotypically heterogeneous. One common phenotype is the hepatocerebral form that manifests in first months of life and causes early death due to liver failure. Liver transplantation (LTx) in not recommended in patients with neurological involvement. Our objective is to raise awareness about the clinical spectrum of early onset liver failure due to MDS. 